\title{
RATIONAL EXPECTATIONS AND THE COURNOT-THEOCHARIS PROBLEM
}

TÖNU PUU

Received 18 April 2006; Accepted 1 May 2006

In dynamic models in economics, often "rational expectations" are assumed. These are meant to show that the agents can correctly foresee the result of their own and the other agents' actions. In this paper, it is shown that this cannot happen in a simple oligopoly model with a linear demand function and constant marginal costs. "Naive expectations," that is, where each agent assumes the other agents to retain their previous period action, are shown to result in a 2-period cycle. However, adapting to the observed periodicity always doubles the actual resulting periodicity. In general, it is impossible for the agents to learn any periodicity except the trivial case of a fixed point. This makes the whole idea of "rational expectations" untenable in Cournot oligopoly models.

Copyright (C) 2006 Tönu Puu. This is an open access article distributed under the Creative Commons Attribution License, which permits unrestricted use, distribution, and reproduction in any medium, provided the original work is properly cited.

\section{Introduction}

In a note in 1959, Theocharis [5] reconsidered the Cournot [2] oligopoly problem, given a linear demand function and constant marginal costs for the competitors. He showed that with three competitors, the Cournot equilibrium becomes neutrally stable, and with four, it becomes unstable. As discussed in [1], the whole argument was antedated 20 years earlier by Palander in $[3,4]$, but as this seems to be totally unknown to the economics profession, we continue referring to the "Theocharis problem." However, both Palander and Theocharis only considered loss of local stability of the Cournot equilibrium point.

In [1], the global dynamics of the model was studied. A linear demand function and constant marginal costs lead to linear reaction functions, which in terms of substance, become nonsense once they result in negative output and profits that are positive due to negative costs dominating over negative revenues. Using nonnegativity constraints, the model necessarily becomes nonlinear, which is needed for the study of the global dynamics. 
The main result of [1] was that the model could asymptotically go to a stable monopoly, a stable duopoly, or else a synchronized 2-period oscillation, in the case of more than three competitors, one where all firms drop out every second period. The outcome solely depended on the marginal costs of the competing firms.

After restating some of these facts, we will now consider that a 2-period cycle (resulting from so-called naive expectations) is easily learnt by the competitors. In particular, if all firms drop out every second period, any firm may attempt to become a monopolist these very periods, and so improve its long-run profit situation.

According to the "rational expectations" hypothesis, all the competitors would learn this 2-periodicity, and so react to conditions, not in the past period, but 2 periods back. As we will see, this changes the resulting periodicity, the cycle becomes 4-periodic. But then, again assuming that all the competitors learnt this, we find that the resulting cycle is doubled, to 8-period, and so forth.

The general issue then is whether it is at all possible for the competitors to learn the right periodicity resulting from their own and their competitors' actions, in accordance with the rational expectations hypothesis. It will be shown that no periodic orbit, except an equilibrium, can be learnt by the agents. In a very vague sense, of course, this reminds of the uncertainty principle in physics. By attempting to measure something, the object of measurement is affected so as to make accurate observation impossible. In the same way, learning the outcome of a dynamical process by the agents involved and adapting to it alters the process so that accurate learning of the process becomes impossible.

\section{Palander (1936/1939) and Theocharis (1959)}

2.1. The model. To restate the basic facts of the global dynamics, let us now specify the model. With linear demand, market price

$$
p:=a-b \sum_{i=1}^{i=n} q_{i}
$$

depends on the total of the supplies of the $n$ competitors. To simplify notation, define total supply

$$
Q:=\sum_{i=1}^{i=n} q_{i}
$$

and "residual supply" (not under the control of the ith competitor)

$$
Q_{i}:=Q-q_{i}, \quad i=1, \ldots, n .
$$

The revenue for the $i$ th competitor hence becomes

$$
R_{i}=\left(a-b\left(Q_{i}+q_{i}\right)\right) q_{i}, \quad i=1, \ldots, n,
$$


whence marginal revenue becomes

$$
\frac{\partial R_{i}}{\partial q_{i}}=a-b Q_{i}-2 b q_{i}, \quad i=1, \ldots, n
$$

Given constant marginal costs, total costs are

$$
C_{i}=c_{i} q_{i}, \quad i=1, \ldots, n
$$

and marginal costs are

$$
\frac{\partial C_{i}}{\partial q_{i}}=c_{i}, \quad i=1, \ldots, n
$$

Equating marginal revenue (2.5) to marginal cost (2.7), we can solve for $q_{i}$ :

$$
q_{i}=\frac{1}{2} \frac{a-c_{i}}{b}-\frac{1}{2} Q_{i}, \quad i=1, \ldots, n,
$$

which can be considered either as a simultaneous system or as a recurrence system.

2.2. Cournot equilibrium. In the first alternative, (2.8), given definitions (2.2) and (2.3), can be solved for the Cournot equilibrium point:

$$
\bar{q}_{i}=\frac{1}{n+1} \frac{a-c_{i}}{b}+\frac{n}{n+1} \frac{\bar{c}-c_{i}}{b}, \quad i=1, \ldots, n,
$$

where for convenience

$$
\bar{c}=\frac{1}{n} \sum_{i=1}^{i=n} c_{i}
$$

Summation of (2.9) gives total output in Cournot equilibrium:

$$
\bar{Q}=\frac{n}{n+1} \frac{a-\bar{c}}{b} .
$$

We see that total Cournot equilibrium output is a fraction of the ratio of maximum price minus average marginal cost to the slope of the inverse demand function. The fraction is one half in the case of monopoly, two thirds in the case of duopoly, and it goes asymptotically to unity as the number of competitors increases.

From (2.11), we can also easily calculate price in the Cournot point:

$$
\bar{p}=a-b \bar{Q}=\frac{1}{n+1} a+\frac{n}{n+1} \bar{c}
$$

which is a weighted average of maximum price and average marginal cost, and hence 
4 Rational expectations and the Cournot-Theocharis problem

positive. Using (2.12) with (2.9), the profits of the individual firms are

$$
\bar{\Pi}_{i}=\left(\bar{p}-c_{i}\right) \bar{q}_{i}=b\left(\frac{1}{n+1} \frac{a-c_{i}}{b}+\frac{n}{n+1} \frac{\bar{c}-c_{i}}{b}\right)^{2}
$$

This squared right-hand side may seem to be always nonnegative, but in case the expression in brackets is negative, we see from (2.9) that $\bar{q}_{i}<0$, which is nonsense. The positive profits then result from negative costs dominating over negative revenues. We will meet this consideration again when constructing the iteration for global dynamics. Hence, in order that a given firm stays in business, we require that $(1 /(n+1))((a-$ $\left.\left.c_{i}\right) / b\right)+(n /(n+1))\left(\left(\bar{c}-c_{i}\right) / b\right)>0$. As the common denominators $(n+1) b>0$, this can be reorganized as follows:

$$
c_{i}<\frac{1}{n+1} a+\frac{n}{n+1} \bar{c}
$$

Only firms with sufficiently low marginal costs will actually stay active in a Cournot equilibrium. Note that from $(2.10), c_{i}$ is one of the terms in $n \bar{c}=\sum_{i=1}^{i=n} c_{i}$, so in order to state a condition for $c_{i}$ in terms of all the $c_{j}, j \neq i$, we later need a further reorganization.

2.3. Reaction functions. As mentioned, we may want to treat (2.8) as a dynamical system. Suppose each firm assumes all the competitors to retain their supplies from the previous period. Then the "best reply" of this firm is

$$
q_{i}(t+1)=\frac{1}{2} \frac{a-c_{i}}{b}-\frac{1}{2} Q_{i}(t), \quad i=1, \ldots, n .
$$

This function (2.15) is usually called the reaction function. In this case, it is linear, due to the underlying linearity of the demand and cost functions, but in general it is not, and then it might be impossible to state in explicit form. As we will see, the reaction function turns nonlinear (piecewise linear) also when we take nonnegativity of output and profits in account.

Note that the derivatives of (2.15),

$$
\frac{\partial q_{i}(t+1)}{\partial Q_{i}(t)}=-\frac{1}{2}, \quad i=1, \ldots, n
$$

are constant, equal to $-1 / 2$, independent of the coordinates of the Cournot equilibrium point.

For further use, take the sum of (2.15) over $i$, and use (2.2), (2.3), and (2.10) to obtain

$$
Q(t+1)=n \frac{1}{2} \frac{a-\bar{c}}{b}-\frac{n-1}{2} Q(t)
$$

Note that this is a divergent process whenever $n>3$. 
2.4. Local stability. The derivatives (2.16) enter the Jacobian matrix of the system in all off-diagonal elements, whereas the diagonal elements are zero. Thus,

$$
J=\left[\begin{array}{rrrr}
0 & -\frac{1}{2} & \cdots & -\frac{1}{2} \\
-\frac{1}{2} & 0 & \cdots & -\frac{1}{2} \\
\vdots & \vdots & \ddots & \vdots \\
-\frac{1}{2} & -\frac{1}{2} & \cdots & 0
\end{array}\right] .
$$

The characteristic equation reads

$$
|J-\lambda I|=\left(\lambda-\frac{1}{2}\right)^{n-1}\left(\lambda+\frac{n-1}{2}\right)=0 .
$$

There are $n-1$ eigenvalues $\lambda_{1, \ldots, n-1}=1 / 2$ and 1 eigenvalue $\lambda_{n}=-(n-1) / 2$. Theocharis concluded that in duopoly, there are two eigenvalues $\lambda_{1}=1 / 2$ and $\lambda_{2}=-1 / 2$, both less than unity in absolute value, so the Cournot equilibrium is stable. In triopoly, the eigenvalues are $\lambda_{1,2}=1 / 2, \lambda_{3}=-1$, so the equilibrium is neutrally stable, with a tendency to endless but stationary oscillation. For $n>3,\left|\lambda_{n}\right|=(n-1) / 2>1$, so the equilibrium becomes unstable. Palander stated the same subject 20 years earlier, though for more general reaction functions.

In his 1939 article, Palander [3] wrote "as a condition for an equilibrium with a certain number of competitors to be stable to exogenous disturbances, one can stipulate that the derivative of the reaction function $f^{\prime}$ must be such that the condition $\left|(n-1) f^{\prime}\right|<1$ holds. If this criterion is applied to, for instance, the case with a linear demand function and constant marginal costs, the equilibria become unstable as soon as the number of competitors exceeds three. Not even in the case of three competitors will equilibrium be restored, rather there remains an endless oscillation." As we see, Palander considers not just linear demand (and hence reaction) functions, but stability of the Cournot equilibrium in general.

As usual, the eigenvalues correspond to a diagonalization of the system, the first $n-1$ associated with differences of the phase variables, the last with the sum of the phase variables.

\section{The global dynamics}

3.1. Nonnegativity of output and profits. It is now time to consider that $q_{i}(t+1)$ cannot become negative. According to (2.15), they must be restricted to

$$
Q_{i}(t) \leq \frac{a-c_{i}}{b}, \quad i=1, \ldots, n
$$

However, there is another fact that we must take in account! Profits must be positive, or the firms will drop out from the market. So, consider expected profits as obtained from 
(2.4) and (2.6),

$$
\Pi_{i}(t+1)=\left(a-c_{i}-b\left(Q_{i}(t)+q_{i}(t+1)\right)\right) q_{i}(t+1), \quad i=1, \ldots, n .
$$

Substituting for $q_{i}(t+1)$ from (2.15), we get

$$
\Pi_{i}(t+1)=\frac{b}{4}\left(\frac{a-c_{i}}{b}-Q_{i}(t)\right)^{2}, \quad i=1, \ldots, n .
$$

The right-hand side of (3.3) being squared, it might seem that we always have a nonnegative profit. However, if (3.1) is not fulfilled, the nonnegativity of profits is due to positive costs dominating over negative revenues, a meaningless case in terms of substance. Hence, the condition for a meaningful nonnegative profit coincides with the condition for nonnegative output, which actually simplifies things.

3.2. The global reaction function. Hence, we reformulate the map as follows:

$$
q_{i}(t+1)=\left\{\begin{array}{ll}
\frac{1}{2} \frac{a-c_{i}}{b}-\frac{1}{2} Q_{i}(t), & Q_{i}(t) \leq \frac{a-c_{i}}{b}, \\
0, & Q_{i}(t)>\frac{a-c_{i}}{b},
\end{array} \quad i=1, \ldots, n .\right.
$$

Numerical experiments with (3.4) indicate that eventually the model can go to steady states of monopoly or duopoly. With three competitors, a permanent oscillation appears, and with four or more, oscillations of period two, where all firms drop out every second period, are the rule. However, any number of competitors may drop out, and the remaining firm(s) may go to Cournot duopoly or even a monopoly state.

\section{Monopoly and duopoly}

We saw that (i) monopoly and duopoly are always stable, and (ii) it is not certain that all the $n$ firms can make positive profits in the Cournot equilibrium point. It may even occur that the relevant condition (2.14), dependent only on the relation between marginal costs, is fulfilled for only one or two firms. If so, the system settles to a stable monopoly or duopoly. The triopoly is a boundary case. We will first check out the possibility for monopoly and duopoly, and then, skipping the boundary case, establish the general global dynamics for $n>3$ remaining competitors.

4.1. Monopoly. In the case of monopoly, where we, without loss of generality, can take the monopolist as number $1,(2.14)$ states that if

$$
c_{i}>\frac{1}{n+1} a+\frac{n}{n+1} \bar{c}, \quad i=2, \ldots, n,
$$

holds, then no competing firm will be able to enter. We can reduce the question to first deal with excluding the possibility of any other firm entering into duopoly. Then $n=2$, and we compare firms 1 and 2, where the second firm, again without loss of generality, 
can represent any of the other firms. We thus get

$$
c_{2}>\frac{1}{3} a+\frac{1}{3}\left(c_{1}+c_{2}\right)
$$

or, rearranging,

$$
c_{2}>\frac{1}{2}\left(a+c_{1}\right)
$$

If this condition does not hold, then the second firm can enter into competition with the monopolist and establish a duopoly. In order for a new firm to make positive profit, and enter in competition with the monopoly, its marginal cost must not exceed a certain number, but note that it does not need to be lower than the marginal cost for the monopolist. If $a=1, c_{1}=0.5$, then the monopoly remains when $c_{2}>0.75$, but transforms into a duopoly when $c_{2}<0.75$-even though the marginal cost of the second firm is $50 \%$ higher than that for the monopolist. As the Cournot point in duopoly is contractive, with eigenvalues $\pm 1 / 2$, the system will approach the Cournot equilibrium.

4.2. Duopoly. Suppose now that one competitor succeeded to intrude the market, and that a duopoly is formed. Again

$$
c_{i}>\frac{1}{n+1} a+\frac{n}{n+1} \bar{c}, \quad i=3, \ldots, n
$$

must hold for the duopoly to remain a duopoly. We can again discuss a possible intrusion of a third firm, $i=3$, as a generic representative for the remaining firms. Thus

$$
c_{3}>\frac{1}{4} a+\frac{1}{4}\left(c_{1}+c_{2}+c_{3}\right)
$$

prevents intrusion. Rearranging,

$$
c_{3}>\frac{1}{3}\left(a+c_{1}+c_{2}\right)
$$

Suppose again $a=1, c_{1}=0.5$, and further $c_{2}=0.6$. Then the nonintrusion condition reads $c_{3}>0.7$, whereas if $c_{3}<0.7$, the third firm will enter. As the eigenvalues are now $1 / 2$, $1 / 2,-1$, the system will become oscillating. However, as illustrated in [1], the new firm can also expel one of the duopolists, so that duopoly remains. This can also happen in the case of monopoly, where a new competitor can throw the previous monopolist out from the market.

\section{The oscillatory case}

5.1. Variable differences. Consider now the general oscillatory case with $n>3$. First let us form the difference $\left(q_{j}(t+1)-q_{i}(t+1)\right)$ according to (2.15), also taking note of $(2.2)$ and (2.3). Hence,

$$
\left(q_{j}(t+1)-q_{i}(t+1)\right)=\frac{1}{2} \frac{c_{i}-c_{j}}{b}+\frac{1}{2}\left(q_{j}(t)-q_{i}(t)\right) .
$$


As we see, this is a contraction, so all differences between variables eventually approach fixed quantities. This relates to what was said above about the first $n-1$ eigenvalues being associated with differences of phase variables.

5.2. The asymptotic process. After any transient has been passed, we have $\left(q_{j}(t)-q_{i}(t)\right)=$ $\left(c_{i}-c_{j}\right) / b$, or better,

$$
q_{j}(t)=q_{i}(t)+\frac{c_{i}-c_{j}}{b}
$$

Summing over $j$, using (2.2) and (2.10), we get

$$
Q(t)=n q_{i}(t)+n \frac{c_{i}-\bar{c}}{b}
$$

or, solving for $q_{i}(t)$,

$$
q_{i}(t)=\frac{\bar{c}-c_{i}}{b}+\frac{1}{n} Q(t), \quad i=1, \ldots, n .
$$

This tells us how each phase variable asymptotically changes with total output of the industry. For the dynamics of $Q(t)$, we have (2.17), which as we saw, unlike the case of (5.1), is oscillatory divergent whenever $n>3$. But this would sooner or later result in a negative $Q(t)$, which cannot happen unless some $q_{i}(t)$ are negative as well, and this cannot happen.

As we are past the transient, (5.4) holds. Further, assume $Q(t)=0$. Then from (5.4),

$$
q_{i}(t)=\frac{\bar{c}-c_{i}}{b}, \quad i=1, \ldots, n
$$

we conclude that $q_{i}(t)>0$ for $c_{i}<\bar{c}$, whereas $q_{i}(t)<0$ for $c_{i}>\bar{c}$. The latter is, however, impossible, so the negative outputs are replaced by zero. If there are any positive outputs, then their aggregate must be positive too, because they cannot be counterbalanced by negative ones, that is, $Q(t)>0$, which contradicts that $Q(t)=0$. Thus, in the asymptotic state, all $q_{i}(t)=0$ whenever $Q(t)=0$.

5.3. The 2-period orbit. Assume now that $Q(t)=0$ in some time period. From (2.17), we then find

$$
Q(t+1)=n \frac{1}{2} \frac{a-\bar{c}}{b}>0,
$$

where positivity holds because maximum price must be higher than all marginal costs, and hence also their average. Further consider also the following iterate, obtained through substituting (5.6) in (2.17) once more, whence

$$
Q(t+2)=-n(n-3) \frac{1}{4} \frac{a-\bar{c}}{b}<0
$$

negative as stated provided that $n>3$. 
This cannot happen, because $Q(t+2)<0$ implies that some $q_{i}(t+2)<0$. Any negative $q_{i}(t+2)$ are, however, replaced by zero. Then, positive $q_{i}(t+2)$ cannot occur, because there are no negative ones that can counterbalance them to make $Q(t+2)<0$. Hence, all $q_{i}(t+2)=0$, which also sum up to $Q(t+2)=0$. We conclude that (i) zero total market output after the passage of two periods again results in a zero output, and (ii) this can only happen through all individual outputs being zero simultaneously as well. From (5.6), we find that the same positive total quantity is produced in the intermediate periods. From (5.4), asymptotically there is a fixed linkage between total output and all the individual outputs, so there definitely is a 2-period cycle where all the firms drop out every second period. This also shows up in any numerical experiment. The condition for this type of 2-period cycle to occur is that the remaining number of firms is $n>3$ as assumed.

\section{Learning the orbit}

6.1. Period doubling. A 2-period orbit is the simplest possible nonconstant orbit, so as soon as the competitors find this out, everybody will adapt to conditions two periods back. They know that their first expectations - that the competitors retain their supplies from the previous period-turned out wrong, and they learned that the competitors' actions recur every second period.

Given this, we can immediately state the recurrence equation

$$
q_{i}(t+2)=\left\{\begin{array}{ll}
\frac{1}{2} \frac{a-c_{i}}{b}-\frac{1}{2} Q_{i}(t), & Q_{i}(t) \leq \frac{a-c_{i}}{b}, \\
0, & Q_{i}(t)>\frac{a-c_{i}}{b},
\end{array} \quad i=1, \ldots, n .\right.
$$

Note that nothing is changed, except the adaptation period. The Cournot equilibrium point is the same, and all considerations about entry and positivity of profits still hold. Recall that we are dealing with $n>3$, that is, marginal costs such that all competitors remain in business.

Obviously, assuming $q_{i}(t)=Q(t)=Q_{i}(t)=0$ and using the above considerations, we conclude that now $q_{i}(t+4)=Q(t+4)=Q_{i}(t+4)=0$ as well. In the intermediate period $t+2$, all quantities will be positive.

So much about periods $t, t+2$, and $t+4$. For periods $t+1, t+3$, and $t+5$, there is another independent 4-period cycle, with alternating zero and positive outputs. How these two processes are combined depends on initial conditions and transients. Anyhow, the resulting process is 4-period, so again, the assumption that the resulting cycle was 2-periodic shows up to be wrong. We can further assume that the competitors learn also this 4-periodicity, only to check out that they actually produce an 8-period cycle, and so it goes.

6.2. Possibility of rational expectations. We may thus pose the following question. Does there exist any periodicity which the competitors can learn, and which coincides with what their behaviour actually results in? Our answer is in the negative. 
Assume there is such a $T$-periodicity. Then, in analogy to (3.4) and (6.1), we have

$$
q_{i}(t+T)=\left\{\begin{array}{ll}
\frac{1}{2} \frac{a-c_{i}}{b}-\frac{1}{2} Q_{i}(t), & Q_{i}(t) \leq \frac{a-c_{i}}{b}, \\
0, & Q_{i}(t)>\frac{a-c_{i}}{b},
\end{array} \quad i=1, \ldots, n .\right.
$$

But under the rational expectations hypothesis, the $T$-periodicity is recognized, so

$$
q_{i}(t+T) \equiv q_{i}(t)
$$

Substituting from (6.3) in (6.2) and taking the first branch of the map,

$$
q_{i}(t)=\frac{1}{2} \frac{a-c_{i}}{b}-\frac{1}{2} Q_{i}(t)
$$

or, using (2.3),

$$
q_{i}(t)=\frac{1}{2} \frac{a-c_{i}}{b}-\frac{1}{2} Q(t)+\frac{1}{2} q_{i}(t)
$$

Finally, rearranging,

$$
q_{i}(t)=\frac{a-c_{i}}{b}-Q(t)
$$

Sum over $i$, use (2.2) and (2.10), and rearrange to solve for

$$
Q(t)=\frac{n}{n+1} \frac{a-\bar{c}}{b}
$$

which equals total supply in Cournot equilibrium, that is, $Q(t)=\bar{Q}$. Further, substituting back in the previous equation, we get

$$
q_{i}(t)=\frac{1}{n+1} \frac{a-c_{i}}{b}+\frac{n}{n+1} \frac{\bar{c}-c_{i}}{b}, \quad i=1, \ldots, n,
$$

which equals the individual supplies in Cournot equilibrium, that is,

$$
q_{i}(t)=\bar{q}_{i}, \quad i=1, \ldots, n
$$

As these relations have to hold for all $t$, we conclude that the only periodic orbit that can be learned is the fixed Cournot point.

Now, we chose the first branch of the reaction function (6.2), so how about the second? From the above reasoning around (2.13), we know that output and profits in Cournot equilibrium are positive, so it is this branch that counts.

By conclusion, in terms of rational expectations, the fixed point is the only periodic orbit that can be learned by the agents. 


\section{Discussion}

The Cournot oligopoly model with linear demand and constant marginal costs was shown by Palander [3] and Theocharis [5] to have an unstable equilibrium whenever the number of competitors becomes larger than 3. The global dynamics of this model can only be studied if nonnegativity constraints for supplies and profits are taken in account. Given this is done, the model displays a 2-period orbit when the competitors react to "naive" expectations, that is, assume that everybody else will supply as in the previous period. Obviously these expectations are not fulfilled.

If the competitors learn the 2-periodicity, and adapt their beahviour to it, the actual periodicity is doubled to 4 , and this goes on with any expectations that try to capture the true periodicity. Learning a periodicity interferes with the process, and alters it. By conclusion, it becomes obvious that the only periodicity that can be learned in the "rational" way is the Cournot fixed point. But as we saw, this fixed point becomes unstable, quite as pointed out by Palander and Theocharis, so it is no alternative with more than 3 competitors.

The natural question then is whether the competitors can learn and adapt to anything else than a periodic orbit. A dynamical system can only be attracted to fixed points or periodic orbits, to quasiperiodic or chaotic orbits, or it can diverge.

Now, in the oligopoly case, divergence indicates misspecification of the model, for instance by neglecting nonnegativity constraints in a linear model. On the other hand, a chaotic trajectory is in principle unpredictable, and a quasiperiodic one requires infinite memory. So, the "rational expectations hypothesis" seems impossible.

It is the conviction of the author that this holds in much more generality, in fact for any reasonable oligopoly model we may construct, and perhaps also in other classes of dynamical models in economics.

\section{References}

[1] J. Cánovas, T. Puu, and M. Ruiz, The Cournot-theocharis problem revisited, to appear in Chaos, Solutions, and Fractals.

[2] A. Cournot, Recherches sur les Principes Mathématiques de la Théorie des Richesses, Hachette, Paris, 1838.

[3] T. F. Palander, Konkurrens och marknadsjämvikt vid duopol och oligopol, Ekonomisk Tidskrift 41 (1939), 124-145, 222-250.

[4] __ Instability in competition between two sellers, Abstracts of Papers Presented at the Research Conference on Economics and Statistics held by the Cowles Commission at Colorado College, Colorado College, General Series no. 208, Studies Series no. 21, 1936.

[5] R. D. Theocharis, On the stability of the Cournot solution on the oligopoly problem, Review of Economic Studies 27 (1959), 133-134.

Tönu Puu: The Center for Regional Science (CERUM), Umeå University, Umeå 90187, Sweden E-mail address: tonu.puu@econ.umu.se 


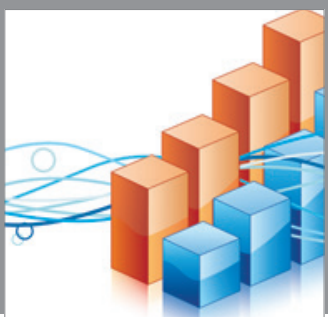

Advances in

Operations Research

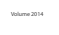

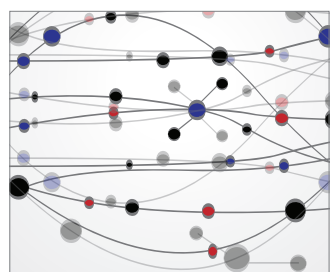

\section{The Scientific} World Journal
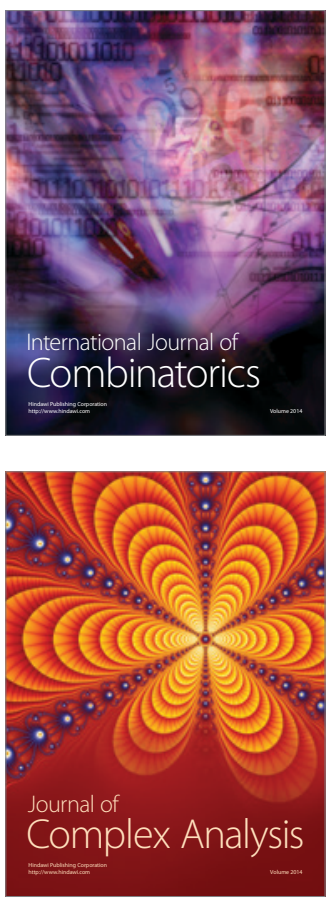

International Journal of

Mathematics and

Mathematical

Sciences
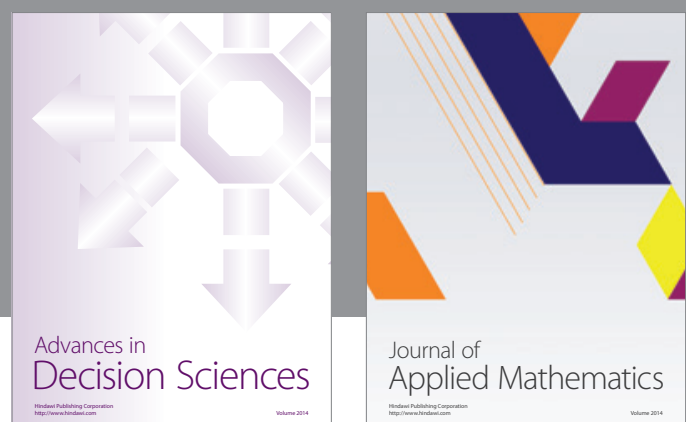

Journal of

Applied Mathematics


Submit your manuscripts at http://www.hindawi.com
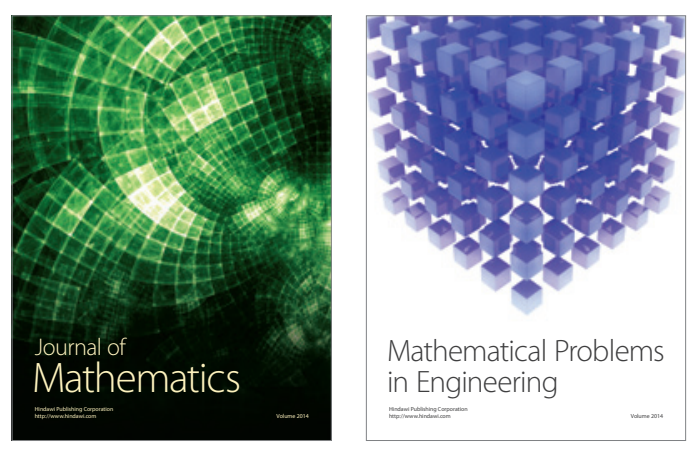

Mathematical Problems in Engineering
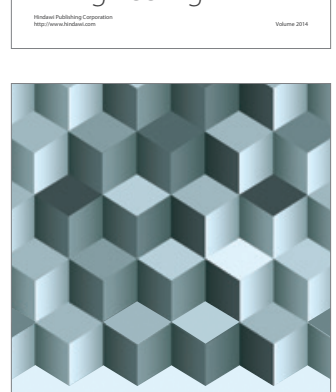

Journal of

Function Spaces
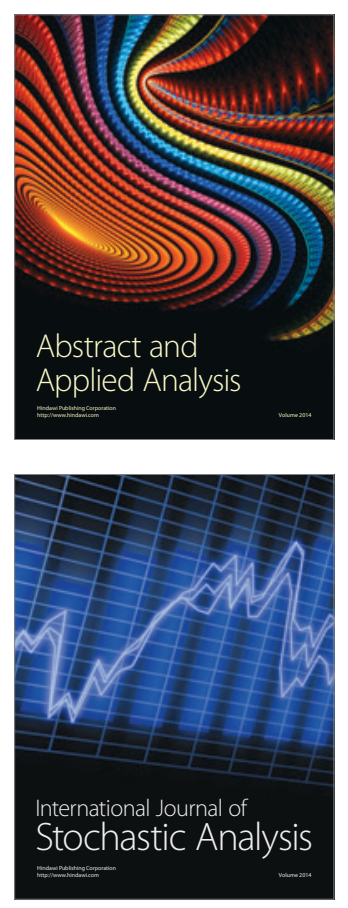

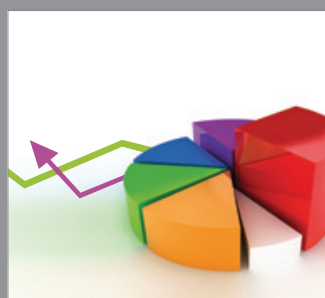

ournal of

Probability and Statistics

Promensencen
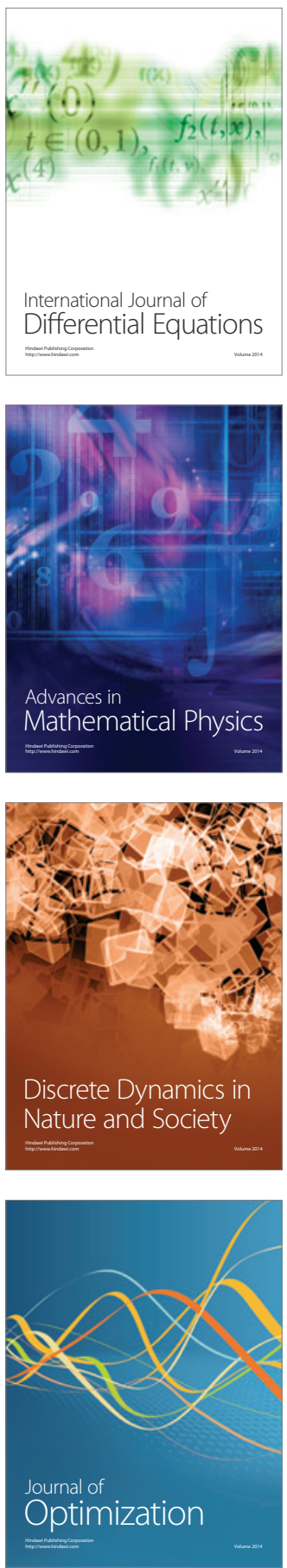